\title{
CRESCIMENTO E CULTIVO DE DIFERENTES ISOLADOS DE PLEUROTUS OSTREATUS (JACQ.: FR.) KUMMER EM SERRAGEM DA CASCA DE COCO
}

\author{
R.H. Marino', L.D. de Abreu², J.B. Mesquita1, G.T. Ribeiro²
}

${ }^{1}$ Universidade Federal deSergipe, Departamento de Engenharia Agronômica, Av. Marechal Rondon, s/nํ, CEP 49100-000, São Cristovão, SE, Brasil, E-mail: rehmarino@yahoo.com

\section{RESUMO}

\begin{abstract}
O objetivo deste trabalho foi avaliar a precocidade na indução de primórdios e no período iniciação da colheita, como também a eficiência biológica de isolados híbridos de Pleurotus ostreatus resistentes ao calor, em serragem da casca de coco suplementada com farelo de arroz e/ ou de trigo, como um substrato alternativo na produção de cogumelos comestíveis, em condições axênicas. A velocidade de crescimento, o vigor, o período de formação de primórdios e da colheita e a eficiência biológica de três isolados de $P$. ostreatus foram avaliados em resposta à suplementação do substrato à base de serragem da casca de coco com 0, 5, 20,30 e 40\% de farelo de trigo e/ou de arroz. É importante ressaltar que a suplementação do substrato à base de serragem da casca de coco com farelos reduziu a relação $\mathrm{C}: \mathrm{N}$ em relação à testemunha e favoreceu a velocidade de crescimento e o vigor micelial, a precocidade na formação de primórdios, a colheita e a eficiência biológica em todos os isolados testados. Dentre os isolados testados, EF60 e POSB destacaram-se como os mais precoces na indução de primórdios e na colheita, como também apresentaram maior eficiência biológica em comparação com o isolado DF39.
\end{abstract}

PALAVRAS-CHAVE: Cogumelos comestíveis, resíduos agrícolas, produção de cogumelos.

\section{ABSTRACT}

GROWTH AND CULTIVATION OF DIFFERENT PLEUROTUS OSTREATUS (JACQ.: FR.) KUMMERSTRAINSONCOCONUT-HUSKSAWDUST.This study evaluated precocity in primordia induction and harvest initiation periods as well as the biological efficiency of heat-resistant hybrid strains of Pleurotus ostreatus using coconut-husk sawdust supplemented with wheat and/or rice bran as an alternative substrate for axenic production of edible mushrooms. The growth rate, vigor, period of primordia formation, harvest initiation period and biological efficiency of three $P$. ostreatus strains were evaluated in response to the supplementation of the coconut-husk sawdust based substrate with $0,5,20,30$ and $40 \%$ of wheat and/or rice bran. The supplementation the coconut-husk sawdust with wheat and/or rice bran reduced the $\mathrm{C}: \mathrm{N}$ rate in comparison with the control group, and increased growth rate, mycelial vigor, precocity of primordia formation and the harvest period, as well as biological efficiency in all strains tested. Among the tested strains, EF60 and POSB were the most precocious in their primordia induction and harvest periods, and were also more biologically efficient than strain DF39.

KEY WORDS: Edible mushrooms, agricultural waste, mushroom production.

\section{INTRODUÇÃO}

Os cogumelos do gênero Pleurotus apresentam grande potencial de cultivo no Brasil em razão de sua maior rusticidade e facilidade de cultivo (EIRA, 2004).

O cogumelo ostra (Pleurotus spp.), natural de florestas tropicais e subtropicais, vem sendo cultivado em vários paises devido sua rusticidade e por ser um eficiente decompositor de resíduos agrícolas e agroindustriais como: esterco, palhas de trigo, arroz, milho, algodão, madeira, bagaço de cana-de-açúcar, resíduos de serrarias, além de muito outros (EIRA , 2004).

Desta forma, o cultivo do cogumelo ostra pode representar uma importante forma de aproveitamento de resíduos agrícolas, contribuindo para geração deempregos, produção dealimentosnutricionalmente saudáveis e minimização de problemas ambientais,

\footnotetext{
${ }^{2}$ Universidade Federal de Sergipe, Núcleo de Engenharia Florestal, São Cristovão, SE, Brasil.
} 
principalmente, pelo depósito de resíduos de lenta decomposição no ambiente (MAZIERO, 1990; STURION, 1994; LeIFA, 2000; FAN et al., 2003; Wu et al., 2004).

Entre os resíduos de potencialidade no Nordeste, para produção de cogumelos, destaca-se a casca do coco. Após o consumo in natura ou industrializado da água de coco, o resíduo, ou seja, a casca do coco é muitas vezes depositada às margens de estradas e rios, o que gera um problema ambiental (Rosa et al., 2001).

A serragem ou pó de coco possui grande porcentagem de lignina (35 a 45\%) e de celulose (23 a 43\%) e uma pequena quantidade de hemicelulose ( 3 a 12\%). Essas características conferem ao substrato grande durabilidade, sendo utilizado para cultivo de plantas de ciclo longo como ornamentais e hortícolas, pois apresenta uma estrutura física vantajosa proporcionando alta porosidade, alto potencial de retenção de umidade, além de ser biodegradável (RosA et al.,2001; CARrijo et al., 2002).

A serragem ou o pó de coco também pode ser utilizado no cultivo de cogumelos comestíveis, como os isolados de Pleurotus spp. (Pedra; Marino, 2006). No entanto, a produtividade é relativamente baixa se compararmos a utilização de outros resíduos lignocelulolíticos, como o bagaço de cana-de-açúcar, resíduos decafé, palha de trigo eserragem deeucalipto (MAZiero, 1990; FAn et al., 2003; BonAtTi et al., 2004; PEDRA; MARINO, 2006).

O objetivo deste trabalho foi avaliar a precocidade na indução de primórdios e no período inicial da colheita, como também a eficiência biológica de isolados híbridos de P. ostreatus resistentes ao calor em serragem da casca de coco suplementada com farelo de arroz e/ ou de trigo, como um substrato alternativo na produção de cogumelos comestíveis, em condições axênicas.

\section{MATERIAL E MÉTODOS}

Os experimentos foram conduzidos na Clínica Fitossanitária do Departamento de Engenharia Agronômica da Universidade Federal deSergipe, São Cristovão, Sergipe, Brasil.

\section{Origem dos isolados}

Foram utilizados os isolados híbridos resistentes ao calor DF39, EF60 e o isolado parental POSB (POS 97/37) provenientes de basidiomas obtidos por doação da Micoteca do Módulo de Cogumelos Comestíveis da Faculdade de Ciências Agronômicas-UNESP, Botucatu, São Paulo.

Os isolados foram repicados, pela transferência de fragmentos do micélio para meio de BDA (batata- dextrose-ágar) e incubados a $25 \pm 3^{\circ} \mathrm{C}$, durante 5 dias (EIRA; MINHONI, 1997)

\section{Preparo do substrato}

O substrato à base de serragem da casca de coco (SCC) foi suplementado com 0,5, 20,30 e 40\% de farelo de arroz e/ou de trigo (Tabela 1) e à mistura adicionou-seágua na quantidadesuficiente para obter aproximadamente $60 \%$ de umidade. Em seguida, $200 \mathrm{~g}$ da mistura foram acondicionados em frascos de $500 \mathrm{~mL}$ e vedado com tampas furadas no centro, preenchido com tampão de algodão e autoclavados por $40 \mathrm{minu}$ tos por duas vezes, com intervalo de $24 \mathrm{~h}$, a $120^{\circ} \mathrm{C}$. Após a autoclavagem e resfriamento do substrato foi realizada a inoculação, em condições assépticas (câmara de fluxo laminar), pela transferência de um disco de $6 \mathrm{~mm}$ de diâmetro proveniente das matrizes multiplicadas conforme descrito no item anterior (PEDRA; MARINO ,2006). A incubação foi à temperatura de $25 \pm 3^{\circ} \mathrm{C}$, por 30 dias. Foram realizadas quatro repetições por isolado.

Tabela 1-Suplementação do substrato à base de serragem da casca de coco (SCC).

\begin{tabular}{crcc}
\hline Tratamento & SCC $^{1}$ & $\begin{array}{c}\text { Farelo de } \\
\text { arroz (FA) }\end{array}$ & $\begin{array}{c}\text { Farelo de } \\
\text { trigo (FT) }\end{array}$ \\
\hline T1 & $100 \%$ & $0 \%$ & $0 \%$ \\
T2 & $95 \%$ & $5 \%$ & $0 \%$ \\
T3 & $95 \%$ & $0 \%$ & $5 \%$ \\
T4 & $80 \%$ & $10 \%$ & $10 \%$ \\
T5 & $70 \%$ & $15 \%$ & $15 \%$ \\
T6 & $60 \%$ & $20 \%$ & $20 \%$ \\
\hline
\end{tabular}

${ }^{1}$ Serragem da casca de coco (SCC) lavada submetida ao processo de compostagem e triturada (dados do fornecedor).

\section{Avaliação do crescimento micelial}

A partir do quarto dia, após a inoculação, os frascos foram avaliados periodicamente e realizadas as medições, cuja metodologia consistiu na inscrição, de três réguas milimetradas, por frasco, cujo ponto 0 da régua milimetrada foi considerado o início do substrato no frasco. As medidas foram realizadas diariamente até a completa colonização do frasco. A diferença entre as medidas consecutivas indica a velocidade de crescimento em $\mathrm{cm} \mathrm{dia}^{-1}$. O vigor foi avaliado pelo critério subjetivo de notas (nota 1 - fracamente adensado; nota 2 - mediamente adensado e nota 3 - fortemente adensado) (PEDRA; MARINO, 2006). 


\section{Avaliação da precocidade na indução de primórdios e da produção}

Após a colonização, os frascos foram submetidos à hidratação e ao choque térmico, após 35 dias da inoculação. Para tanto, retirou-se a tampa dos frascos e foi acrescentada uma lâmina d'água destilada. Em seguida, os frascos foram acondicionados em um refrigerador à temperatura de $8 \pm 2^{\circ} \mathrm{C}$, durante $4 \mathrm{~h}$ (EIRA; MinHonI, 1997). Após a hidratação e choque térmico, os frascosforam transferidos para o ambiente rústico de frutificação. Foram avaliados: o período de indução de primórdios (em dias), o período de início da colheita (em dias) e a eficiência biológica (gramas de cogumelos frescos/grama de substrato fresco $x$ 100; em \%), por um fluxo de produção.

\section{Determinação da relação carbono: nitrogênio do substrato}

A determinação da relação carbono:nitrogênio (C:N) foi realizada segundo a metodologia deSANCHES (1999). Para tanto, o teor de carbono foi determinado pela digestão de $100 \mathrm{mg}$ de substrato seco e moído em dicromato de potássio e ácido sulfúrico concentrado e, em seguida, titulado com solução de sulfato ferroso amoniacal. A determinação de nitrogênio foi realizada pelo Método de Kjeldahl (SAnChes, 1999). Para tanto, utilizou-se três amostras do substrato preparado, conforme cada tratamento e previamente autoclavado. As amostras foram retiradas antes e depois da colonização pelos isolados $P$. ostreatus.

A relação C: $N$ foi determinada segundo a fórmula abaixo:

$$
C: N=\frac{\% C}{\% N}
$$

\section{Análise estatística}

Os resultados da velocidade de crescimento $(\mathrm{cm}$ dia $^{-1}$ ), vigor (notas), período de indução de primórdios (dias), o período de início da colheita (dias) e a eficiência biológica (\%) foram submetidos à Análise de Variância (ANOVA) e, para comparação das médias foi utilizado o teste de Tukey a $5 \%$ de probabilidade.

\section{RESULTADOS E DISCUSSÃO}

\section{Crescimento micelial}

A produção de cogumelos depende da rápida colonização e formação de micélio vigoroso. Estes fatores refletem a capacidade do substrato ser colonizado e estão diretamente relacionados com a habili- dade dos isolados do cogumelo P.ostreatus em decompor o substrato. A rápida colonização é favorável, pois reduz a perda de substrato por contaminação e também permite curto período de incubação e frutificação.

Considerando os resultados obtidos, os isolados de $P$. ostreatus testados foram capazes de degradar e colonizar o substrato SCC (Tabela 2).

Tabela 2 - Dados médios de velocidade de crescimento $\left(\mathrm{cm} \mathrm{dia}{ }^{-1}\right)$ dos isolados de Pleurotusostreatus cultivados em serragem da casca de coco (SCC) suplementada com 0,5 , 20,30 e $40 \%$ de farelo de arroz e/ ou de trigo.

\begin{tabular}{lcc}
\hline Isolados & $\begin{array}{c}\text { Velocidade de } \\
\text { crescimento }\left(\mathrm{cm} \mathrm{dia}^{-1}\right)\end{array}$ & $\begin{array}{c}\text { Vigor micelial } \\
\left(\text { notas }^{1}\right)\end{array}$ \\
\hline DF39 & $0,69 \mathrm{a}$ & $2,33 \mathrm{a}$ \\
EF60 & $0,67 \mathrm{a}$ & $2,33 \mathrm{a}$ \\
POS B & $0,63 \mathrm{a}$ & $2,33 \mathrm{a}$ \\
\hline
\end{tabular}

${ }^{1}$ Vigor-critério subjetivo (notas): 1 - fracamenteadensado; 2 - mediamente adensado; 3 - fortemente adensado;

${ }^{2}$ Médias seguidas pela mesma letra não diferem estatisticamente entre si pelo Teste de Tukey a 5\% de probabilidade.

A velocidade média de crescimento foi de $0,66 \mathrm{~cm}$ $\mathrm{dia}^{-1}$, sem diferença significativa entre os isolados testados, valores estes similares ao encontrado por PedRa;Marino(2006), no cultivo de outros isolados de P. ostreatus, também resistentes ao calor, neste mesmo tipo de substrato. Por outro lado, o cultivo dos isolados EF60, DF39 e POSB em meio de cultura à base de batata-dextrose-ágar resultou em uma velocidade média de crescimento de $0,75 \mathrm{~cm} \mathrm{dia}^{-1}$ (Menezes et al., 2005), demonstrando o efeito da composição química do meio de cultura no crescimento micelial, como também observados por PHILLIPOUssis et al. (2001).

Além disso, a casca de coco é um resíduo que apresenta elevada concentração de taninos, composto tóxico responsável pela redução do crescimento de plantas e microrganismos (Rosa et al., 2001). O efeito do tanino sobre o crescimento de cogumelos comestíveis como o P. ostreatus foi relatado por LeIFA (1999), (2000) e FAN et al. (2003), em que citou a redução do crescimento de alguns isolados de Pleurotus quando cultivados em resíduos de café. Neste experimento, a lavagem da fibra da casca de coco, antes da obtenção da serragem, pode ter favorecido a colonização dos isolados de Pleurotus ostreatus devido a retirada de parte do tanino e/ou de outros compostos tóxicos presentes no substrato.

Por sua vez, SALMONES et al. (2005) mencionaram que o crescimento vegetativo de Lentinula edodes, 
Agaricus bisporus e isolados dePleurotus foi favorecido pela atividade enzimática de lacase, responsável pela redução da concentração de compostos fenólicos e, consequentemente, acelerou a colonização, aumentou a densidade ou vigor micelial e reduziu os riscos de contaminação.

PEDRA; MARINO (2006) relataram que suplementação com 20 e $40 \%$ de farelos de trigo ou de arroz aumentou a velocidade de crescimento micelial e a densidade micelial, tal como observado neste experimento a partir de 5\% de suplementação, sem diferença significativa entre a utilização de farelo de arroz ou de trigo (Tabela 2) e (Figs. 1 e 2).

A adição de farelos à serragem de madeira é relatada na literatura como um fator estimulante ao crescimento devidoa presença de carboidratos, aminoácidos e minerais prontamente assimiláveis pelo fungo (FASIDI; KADIRI, 1993; RoYse, 1996).SONG et al. (1989) citaram que a suplementação com farelo de arroz forneceu altos teores de lipídeos e favoreceu o crescimento micelial mais vigoroso de L. edodes.

A
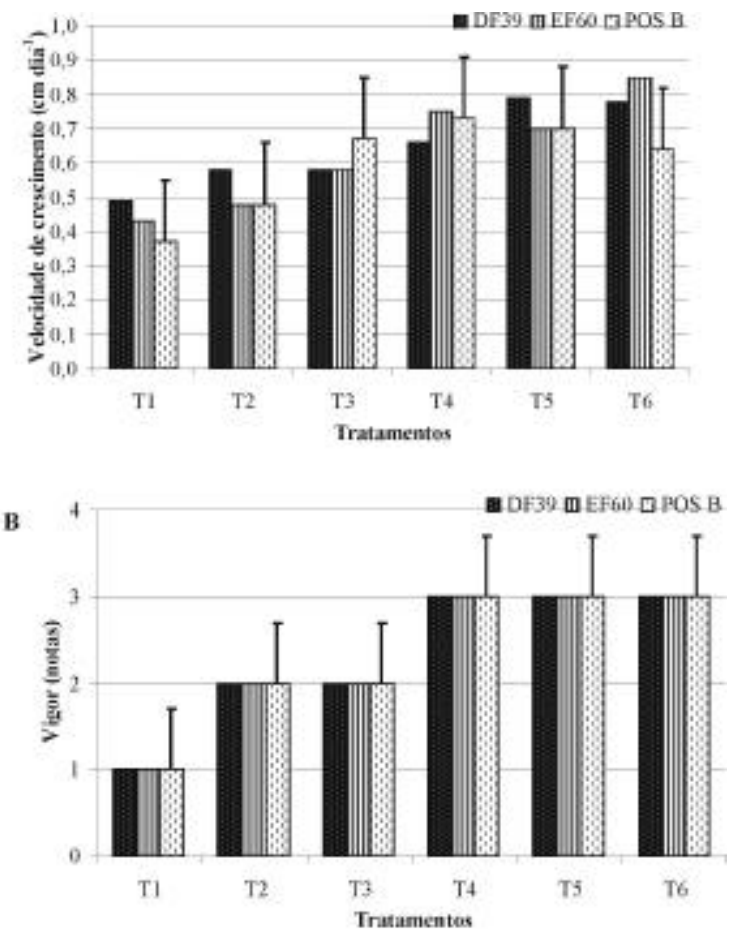

Fig. 1 - Crescimento micelial ${ }^{1}$ dos isolados Pleurotus ostreatus $^{2}$ cultivados em serragem da casca de coco suplementada com farelos ${ }^{3}$.

${ }^{1} \mathrm{~A}$ - Velocidade crescimento média $\left(\mathrm{cm} \mathrm{dia}^{-1}\right)$ e B - Vigor micelial (notas): 1 -fracamente adensado; 2 -mediamente adensado; 3 - fortemente adensado;

2 Isolados: DF39, EF60 e POSB;

${ }^{3}$ Tratamentos: $\mathrm{T} 1=$ controle; $\mathrm{T} 2=5 \%$ de farelo de arroz (FA); T3 $=5 \%$ de farelo de trigo (FT); T4 $=10 \% \mathrm{FA}+10 \%$ FT; $\mathrm{T} 5=15 \% \mathrm{FA}+15 \%$ FT e T6 $=20 \% \mathrm{FA}+20 \% \mathrm{FT}$.

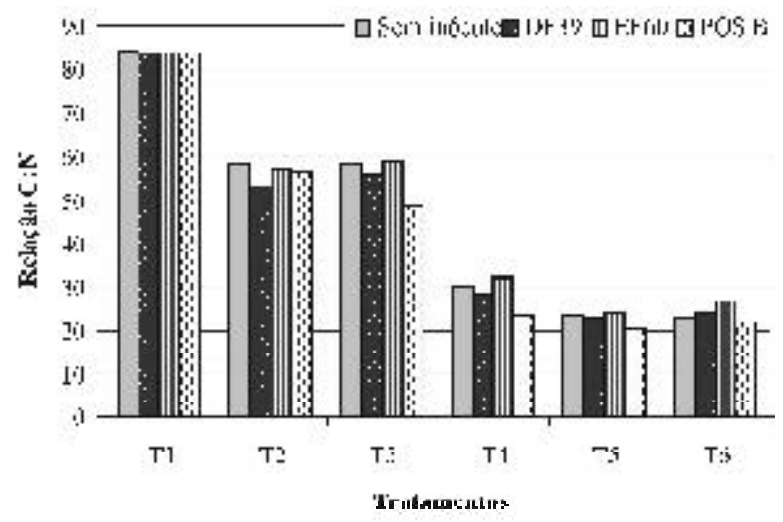

Fig. 2 - Relação C:N da serragem da casca de coco nos diferentes tratamentos ${ }^{1}$ antes e depois da colonização do substrato pelo micélio dos isolados dePleurotusostreatus ${ }^{2}$. ${ }^{1}$ Tratamentos: $\mathrm{T} 1=$ controle; $\mathrm{T} 2=5 \%$ de farelo de arroz (FA); T3 $=5 \%$ de farelo de trigo (FT); T4 $=10 \% \mathrm{FA}+10 \%$ FT; $55=15 \%$ FA $+15 \%$ FT e T6 $=20 \%$ FA $+20 \%$ FT. ${ }^{2}$ Isolados: DF39, EF60 e POS B.

É importante ressaltar que a suplementação de substratos lignocelulósicos pode alterar a relação $\mathrm{C}: \mathrm{N}$ do substrato e influenciar o crescimento micelial (SING; Verma, 1996). Neste experimento, a relação $\mathrm{C}: \mathrm{N}$ da serragem da casca de coco sem suplementação apresentou valor médio de 84 , valor inferior ao citado porSANCHES(1999), provavelmente devido à procedência, ao sistema de cultivo, às condições climáticas e variedades de coco utilizadas na fabricação da serragem. É importante ressaltar que a suplementação do substrato à base de serragem da casca de coco com farelos reduziu a relação $\mathrm{C}: \mathrm{N}$ em relação à testemunha e favoreceu o crescimento e vigor micelial dos três isolados testados (Fig. 2).

\section{Precocidade na indução de primórdios e produção}

O período médio de indução de primórdios foi de 19,2 dias, com inicio da colheita, em média, após 4,6 dias da formação dos primórdios e com uma eficiência biológica média de 5,9\% dos isolados testados em SCC (Tabela 3).

É importante enfatizar que a suplementação de 20 a $40 \%$ com farelo de arroz ou de trigo em relação ao tratamento controle e os isolados testados favoreceu a precocidadena indução de primórdios e a eficiência biológica (Tabelas 3 a 6), tal como também observado por PEDRA; MARINO (2006), B ANIK; NANDI (2004) eWANG et al. (2001).

Da mesma forma que observado por PEDRA; MARINO(2006), a utilização de farelo de arroz ou de trigo não influenciou na precocidade de indução de primórdios e na eficiência biológica (Tabelas 4 a 6). 
Tabela 3 - Dados médios do período de formação de primórdios, colheita, produção e eficiência biológica dos isolados de Pleurotus ostreatus cultivados em serragem da casca de coco (SCC) suplementado com 0, 5, 20, 30 e $40 \%$ de farelo de arroz e/ou de trigo.

\begin{tabular}{lccc}
\hline Isolados & \multicolumn{2}{c}{ Período de } & Eficiência biológica $^{1}$ (\%) \\
\cline { 2 - 4 } & Indução de primórdios (dias) & Início da colheita (dias) & \\
\hline DF 39 & $20,5 \mathrm{~b}^{2}$ & $4,7 \mathrm{~b}$ & $5,4 \mathrm{~b}$ \\
EF 60 & $18,5 \mathrm{a}$ & $4,5 \mathrm{a}$ & $6,3 \mathrm{a}$ \\
POS B & $18,5 \mathrm{a}$ & $4,5 \mathrm{a}$ & $6,0 \mathrm{a}$ \\
\hline
\end{tabular}

${ }^{1}$ Base úmida/úmida;

${ }^{2}$ Médias seguidas pela mesma letra, na coluna, não diferem estatisticamente entre si pelo Teste de Tukey a 5\% de probabilidade.

Tabela 4 - Dados médios do período de formação de primórdios, colheita, produção e eficiência biológica do isolado DF39 de Pleurotus ostreatus cultivado em serragem da casca de coco (SCC) suplementado com farelos.

\begin{tabular}{cccc}
\hline Tratamentos $^{1}$ & \multicolumn{2}{c}{ Período de } & Eficiência biológica $^{2}(\%)$ \\
\cline { 2 - 4 } & Indução de primórdios (dias) & Início da colheita (dias) & \\
\hline T1 & $\mathrm{Np}^{3}$ & $\mathrm{~Np}$ & $\mathrm{~Np}$ \\
$\mathrm{~T} 2$ & $\mathrm{~Np}$ & $\mathrm{~Np}$ & $\mathrm{~Np}$ \\
$\mathrm{~T} 3$ & $\mathrm{~Np}$ & $\mathrm{~Np}$ & $\mathrm{~Np}$ \\
$\mathrm{~T} 4$ & 20,5 & 4,7 & 5,4 \\
$\mathrm{~T} 5$ & $\mathrm{~Np}$ & $\mathrm{~Np}$ & $\mathrm{~Np}$ \\
$\mathrm{~T} 6$ & $\mathrm{~Np}$ & $\mathrm{~Np}$ & $\mathrm{~Np}$
\end{tabular}

${ }^{1}$ Tratamentos: $\mathrm{T} 1$ = controle; $\mathrm{T} 2$ =5\% de farelo de arroz (FA); T3 = 5\% de farelo de trigo (FT); T4 = 10\% FA+ 10\% FT; T5

$=15 \% \mathrm{FA}+15 \% \mathrm{FT}$ e $\mathrm{T} 6=20 \% \mathrm{FA}+20 \% \mathrm{FT}$.

${ }^{2}$ Base úmida/úmida;

${ }^{3} \mathrm{~Np}$ - não produziu cogumelos.

Tabela 5 - Dados médios do período de formação de primórdios, colheita, produção e eficiência biológica do isolado EF60 de Pleurotus ostreatus cultivado em serragem da casca de coco (SCC) e suplementado com farelos.

\begin{tabular}{cccc}
\hline Tratamentos $^{1}$ & \multicolumn{2}{c}{ Período de } & Eficiência biológica $^{2}(\%)$ \\
\cline { 2 - 4 } & Indução de primórdios (dias) & Início da colheita (dias) & \\
\hline $\mathrm{T} 1$ & $\mathrm{~Np}^{3}$ & $\mathrm{~Np}$ & $\mathrm{~Np}$ \\
$\mathrm{~T} 2$ & $\mathrm{~Np}$ & $\mathrm{~Np}$ & $\mathrm{~Np}$ \\
$\mathrm{~T} 3$ & $\mathrm{~Np}$ & $\mathrm{~Np}$ & $\mathrm{~Np}$ \\
$\mathrm{~T} 4$ & $18,7 \mathrm{a}^{4}$ & $4,7 \mathrm{a}$ & $3,8 \mathrm{~b}$ \\
$\mathrm{~T} 5$ & $18,4 \mathrm{a}$ & $4,5 \mathrm{a}$ & $7,5 \mathrm{a}$ \\
$\mathrm{T} 6$ & $18,3 \mathrm{a}$ & $4,3 \mathrm{a}$ & $7,5 \mathrm{a}$ \\
\hline
\end{tabular}

${ }^{1}$ Tratamentos: $\mathrm{T} 1$ = controle; $\mathrm{T} 2=5 \%$ de farelo de arroz $(\mathrm{FA}) ; \mathrm{T} 3=5 \%$ de farelo de trigo $(\mathrm{FT}) ; \mathrm{T} 4=10 \% \mathrm{FA}+10 \% \mathrm{FT} ; \mathrm{T} 5$

$=15 \% \mathrm{FA}+15 \%$ FT e T $6=20 \% \mathrm{FA}+20 \%$ FT.

${ }^{2}$ Base úmida/úmida;

${ }^{3} \mathrm{~Np}$ - não produziu cogumelos;

${ }^{4}$ Médias seguidas pela mesma letra, na coluna, não diferem estatisticamente entre si pelo Teste de Tukey a 5\% de probabilidade.

Dentre os isolados testados, EF60 e POSB destacaram-se como os mais precoces na indução de primórdios e na colheita, como também apresentaram maior eficiência biológica em comparação com o iso- lado DF39 (Tabela 3). O isolado DF50 de P.ostreatus, também resistente ao calor, apresentou período médio de indução de primórdios de 33,80 dias em serragem da casca de coco (Pedra; Marino, 2006). 
Tabela 6 - Dados médios do período de formação de primórdios, colheita, produção e eficiência biológica do isolado POS B de Pleurotus ostreatus cultivado em serragem da casca de coco (SCC) suplementado com farelos.

\begin{tabular}{cccc}
\hline Tratamentos $^{1}$ & \multicolumn{2}{c}{ Período de } & Eficiência biológica ${ }^{2}(\%)$ \\
\cline { 2 - 4 } & Indução de primórdios (dias) & Início da colheita (dias) & \\
\hline T1 & $\mathrm{Np}^{3}$ & $\mathrm{~Np}$ & $\mathrm{~Np}$ \\
$\mathrm{~T} 2$ & $\mathrm{~Np}$ & $\mathrm{~Np}$ & $\mathrm{~Np}$ \\
$\mathrm{~T} 3$ & $\mathrm{~Np}$ & $\mathrm{~Np}$ & $\mathrm{~Np}$ \\
$\mathrm{~T} 4$ & $20,0 \mathrm{a}^{4}$ & $5,4 \mathrm{a}$ & $5,8 \mathrm{a}$ \\
$\mathrm{T} 5$ & $18,5 \mathrm{a}$ & $4,5 \mathrm{~b}$ & $6,3 \mathrm{a}$ \\
$\mathrm{T} 6$ & $17,0 \mathrm{a}$ & $3,6 \mathrm{c}$ & $5,8 \mathrm{a}$ \\
\hline
\end{tabular}

${ }^{1}$ Tratamentos: $\mathrm{T} 1=$ controle; $\mathrm{T} 2=5 \%$ de farelo de arroz $(\mathrm{FA}) ; \mathrm{T} 3=5 \%$ de farelo de trigo $(\mathrm{FT}) ; \mathrm{T} 4=10 \% \mathrm{FA}+10 \% \mathrm{FT} ; \mathrm{T} 5$ $=15 \% \mathrm{FA}+15 \% \mathrm{FT}$ e T6 $=20 \% \mathrm{FA}+20 \% \mathrm{FT}$.

${ }^{2}$ Base úmida/úmida; ${ }^{3} \mathrm{~Np}$ - não produziu cogumelos; ${ }^{4}$ Médias seguidas pela mesma letra, na coluna, não diferem estatisticamente entre si pelo Teste de Tukey a $5 \%$ de probabilidade.

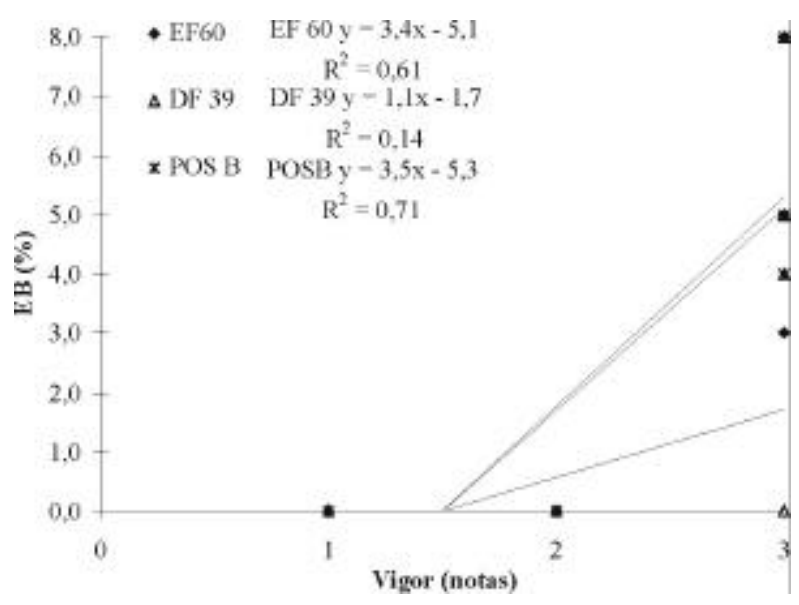

Fig. 3 - Análise de correlação entre os parâmetros eficiência biológica e vigor dos isolados Pleurotus ostreatus cultivados em serragem da casca de coco suplementada com farelos.

MARINO (2002) citou que os isolados DF39, EF60 e POSB induziram primórdios em substrato à base de serragem deEucalyptus sp.com $20 \%$ defarelo de trigoede arroz, em média, aos 6, 4 e 8 dias após o choque térmico. Este comportamento evidencia o efeito do substrato no desenvolvimento dos três isolados de P.ostreatus, talcomo observado por MAZIERO (1990) no cultivo de Pleurotus em polpa de café, com alta concentração de taninos.

Da mesma forma, SALMONES et al.(2005) relataram que dois isolados de $P$. ostreatus cultivados em polpa de café e em palha de trigo induziram primórdios, em média, aos 28 e 18 dias, respectivamente. Estes autores evidenciam que a presença decompostos fenólicos na polpa de café influenciou negativamentena precocidade dos isolados de Pleurotus spp. testados, como observado nos resíduos de café (LEIFA, 1999, 2000;FAN et al., 2003) e na casca de coco (RosA et al., 2001).

Pela análise de regressão dos dados de vigor e da eficiência biológica observa-se que os isolados com crescimento micelial vigoroso foram os mais produtivos (Fig. 3). Este comportamento deve ser em resposta à suplementação que disponibilizou nutrientes ricos em carbono e nitrogênio prontamente utilizável pelo fungo (FASIDI;KADIRI, 1993;RoySE, 1996;SONG etal.,1989), reduziu a relação C:N (Fig. 2) e, consequentemente, favoreceu a precocidadena indução de primórdios dos isolados testados, principalmente a partir de $20 \%$ de suplementação com farelos (Fig. 3; Tabelas 4 a 6).

É importante ressaltar, que não houve indução de primórdios nos tratamentos T1 a T3 (0 a 5\% de suplementação) em todos os isolados, e nos tratamentos T5 e T6 (30 e 40\% de suplementação) com o isolado POSB por até 35 dias após o término da colonização (Tabelas 4 a 6 ).

O cultivo dos isolados POSB, DF39 e EF60 em serragem de eucalipto suplementado com $20 \%$ de farelo de arroz e de trigo resultou em uma eficiência biológica média de 23,3,20,7 e 38,4\% , respectivamente, em dois ciclos de produção (MARINo et al., 2003). Neste experimento, com o cultivo destes isolados em serragem da casca de coco, a eficiência biológica foi, em média, de 6,0, 5,4 e 6,3, respectivamente, dos isolados POSB, DF39 e EF60. A redução da eficiência biológica também foi observada por SALMONES et al. (2005) entre os isolados de Pleurotus spp. cultivados em polpa de café, devido a presença de compostos fenólicos, influencia na precocidade na indução de primórdios e no início da colheita.

Outro fator a ser consideradoéa influência do tipo de substrato na qualidade dos basidiomas produzidos. LeIFA (1999) observou alteração morfológica dos basidiomas de Pleurotus cultivados em borra de café. Já Thomas etal.(1998), no cultivo dePleurotussajor-caju em folhas e ramos de coqueiro, relataram que não houve deformações dos cogumelos produzidos neste substrato, como observado por PEDRA; MARINO (2006) e neste experimento (Fig. 4). 


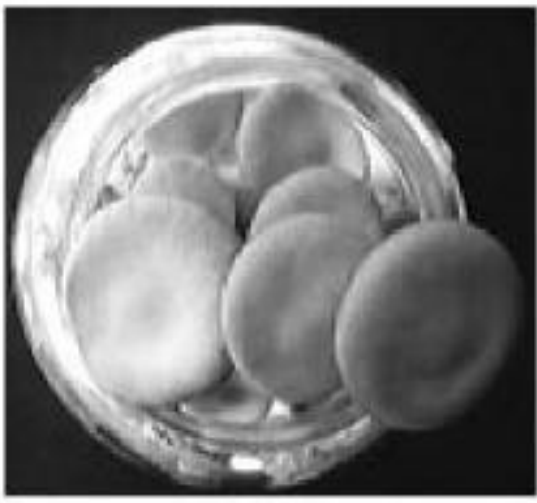

DF 50

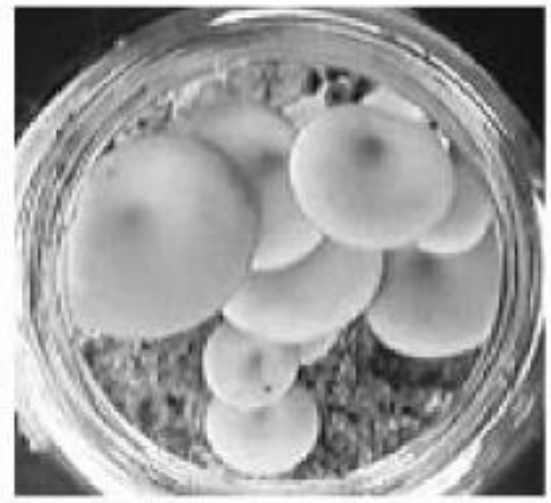

EF 60

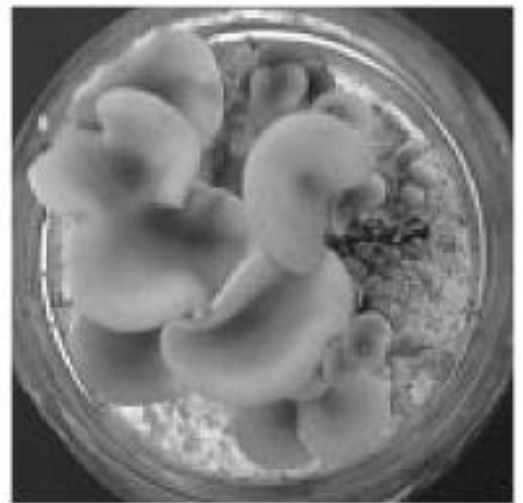

POS B

Fig. 4 - Basidiomas dos isolados dePleurotus ostreatus cultivados em serragem da casca decoco suplementada com farelos.

Portanto, a suplementação da serragem da casca de coco favoreceu o crescimento e a produção dos três isolados testados em relação à testemunha.

\section{AGRADECIMENTOS}

Os autores agradecem à Fundação de Amparo à Pesquisa do Estado de Sergipe (FAP-SE) pelo financiamento e ao Conselho Nacional de Desenvolvimento Científico e Tecnológico(CNPq)eUniversidadeFederal de Sergipe pelas bolsas de estudos.

\section{REFERÊNCIAS}

BANIK, S.; NANDI, R. Effect supplementation of rice stains with biogas residual slurry manure on the yield protein and mineral contents of oyster mushroom. Industrial Crops and Products, v.20, p.311-319, 2004.

BONATTI, M.; KARNOPP, P.; SOARES, H.M.; FURLAN, S.A. Evaluation of Pleurotus ostreatus and Pleurotus sajorcaju nutritional characteristics when cultivated in different lignocellulosic wastes. Food Chemestry, v.88, p.425-428, 2004.

CARRIJO, O.A.; LIZ, R.S.; MAKISHIMA, N. Fibra de coco verde como substrato agrícola. Horticultura Brasileira, v.20, n.4, p.533-535, 2002.

EIRA, A.F. Fungos comestíveis. In: ESPÓSITO, E.; AZEVEDO, J.L. (Ed.). Fungos: uma introdução à biologia, bioquímica e biotecnologia. Caxias do Sul: Educs, 2004. 510p. Capítulo 12.

EIRA, A.F.; MINHONI, M.T.A. Manual teórico-prático do cultivo de cogumelos comestíveis. Botucatu: Fundação de Pesquisa Agropecuária e Florestais, 1997. 75p.

FAN, L.; SOCCOL, A.T.; PANDEY, A.; SOCCOL, C.R. Cultivation of Pleurotus mushrooms on Brazilian coffee husk and effects of caffeine and tannic acid. Micologia Aplicada International, v.15, p.15-21, 2003.

FASIDI, I.O.; KADIRI, M. Use of agricultural wastes for the cultivation of Lentinus subnudus (Poliporales: Polyporaceae) in Nigeria. Revista de Biologia Tropical, v.41, n.3, p.411-415, 1993.

LEIFA, F. Produção de fungo comestível do gênero Pleurotus em bio-resíduos da agroindústria do café. 1999. 92p. Dissertação (Mestrado) - Universidade Federal do Paraná, Curitiba, 1999.

LEIFA, F.; PANDEY, A.;SOCCOL, C.R. Production of mushrooms on Brazilian coffee industry residues. In: SERA, T.; SOCCOL, C.R.; PANDEY, A. (Ed.). Coffee biotechnology and quality. Dordrecht: Kluwer Academic Press, 2000. p.427-436.

MARINO, R.H. Melhoramento genético de Pleurotus ostreatus visando o cultivo axênico de linhagens resistentes ao calor. 2002. 109p. Tese (Doutorado em Biotecnologia) - Instituto de Química, Universidade Estadual Paulista, Araraquara, 2002.

MARINO, R.H.; EIRA, A.F.; KURAMAE, E.E.; QUEIROZ, E.C. Morphomolecular characterization of Pleurotus ostreatus (Jacq. Fr.) Kummer strains in relation to luminosity and temperature of fructification. Scientia Agricola, v.60, n.3, p. 419-424, 2003.

MAZIERO, R. Substratos alternativos para o cultivo de Pleurotus spp. 1990. 136p. Dissertação (Mestrado em Ciências Biológicas) - Instituto de Biociências, Universidade São Paulo, São Paulo, 1990.

MENEZES, B.C.; ARAÚJO, P.H.O; MARINO, R.H. Avaliação do crescimento micelial do cogumelo Pleurotus spp. em diferente concentrações de herbicida Padron. In: REUNIÃO ANUAL DA SBPC, 57., 2005, Fortaleza, CE. Resumos. Fortaleza: 2005. p.63. 
PEDRA, W.N.; MARINO, R.H. Cultivo axênico de Pleurotus spp. em serragem da casca de coco (Cocos nucifera Linn.) suplementada com farelo de arroz e/ou de trigo. Arquivos do Instituto Biológico, São Paulo, v.73, n.2, p.219-225, 2006.

PHILIPPOUSSIS, A.; ZERVAKIS, G.;

DIAMANTOPOULOU, P. Bioconversion of agricultural lignocelullosic wastes through the cultivation of the edible mushrooms Agrocybe aeregita, Volvariella volvaceae and Pleurotus spp. World Journal of Microbiology \& Biotechnology, v.17, p.191-200, 2001.

ROSA, M.F.; SANTOS, F.J.S.; MONTENEGRO, A.A.T.; ABREU, F.A.P.; CORREIA, D.; ARAÚJO, F.B.S.; NORÕES, E.R.V. Caracterização do pó da casca de coco verde usado como substrato agrícola. Comunicado Técnico Embrapa Agroindústria Tropical, n.54, p.1-6, 2001.

ROYSE, D.J. Specialty mushrooms. In: JANICK, J. (Ed.). Process in new crops. Arlingon: ASH Press, 1996. p.464-475.

SALMONES, D.; MATA, G.; WALISZEWSKI, K.N. Comparative culturing of Pleurotus spp. on coffee pulp and wheat straw: biomass production and substrate biodegradation. Bioresource Technology, v.96, p.537-544, 2005.

SANCHES, F.P. Propriedades y características de los substratos. Turba y fibra de coco. In: FERNÁNDEZ, M.F.; GÓMEZ, I.M.C. (Ed.). Cultivos in suelo II. 2. ed. Almería: Dirección Gen. de Investigación y Formación Agraria; FIAPA; Caja Rural de Almería, 1999. p.65-92.
SINGH, T.G.; VERMA, R.N. Studies on carbon and nitrogen of Lentinula lateritia (Berk.) Pegler strains from northeastern India. In: INTERNATIONAL

CONFERENCE MUSHROOM BIOLOGY AND

MUSHROOM PRODUCTS, 2., 1996, Pennsylvania.

Proceedings. Pennsylvania: 1996. p.345-354.

SONG, C.H.; CHO, K.Y.; NAIR, N.G. Growth

stimulation and lipid synthesis in Lentinus edodes. Mycologia, v.81, n.4, p.514-522, 1989.

STURION, G.L. Utilização da folha da bananeira como substrato para o cultivo de cogumelos comestiveis (Pleurotus spp.). 1994. 147p. Dissertação (Mestrado em ciências e Tecnologia de Alimentos) - Universidade de São Paulo, Piracicaba, 1994.

THOMAS, G.V.; PRABHU, S.R.; REENY, M.Z.;

BOPAIAH, B.M. Evaluation of lignocellulosic biomass from coconut palm as substrate for cultivation of Pleurotus sajor-caju (Fr.) Singer. World Journal of Microbiology \& Biotecnology, v.14, p. 879-882, 1998.

WANG, D.; SAKODA, A.; SUZUKI, M. Biological efficiency and nutritional value of Pleurotus ostreatus cultivated on spent beer grain. Bioresource Technology, v.78, p.293-300, 2001.

WU, J.Z.; CHEUNG, P.C.K.; WONG, K.H.; HUANG, N.L. Studies on submerged fermentation of Pleurotus tuber-regium (Fr.) Singer. Part 2: Effect of carbon-tonitrogen ratio of the culture medium on the content and composition of the mycelial dietary fiber. Food Chemistry, v.85, p.101-105, 2004.

Recebido em 28/2/07

Aceito em 12/3/08 